\title{
The influence of MOVPE growth conditions on the shell of core-shell GaN microrod structures
}

\author{
Tilman Schimpke , Adrian Avramescu , Andreas Koller , Amalia Fernando-Saavedra , Jana Hartmann \\ Johannes Ledig , Andreas Waag , Martin Strassburg , Hans-Jürgen Lugauer
}

\begin{abstract}
A B S T R A C T
A core-shell geometry is employed for most next-generation, three-dimensional opto-electric devices based on III-V semiconductors and grown by metal organic vapor phase epitaxy (MOVPE). Controlling the shape of the shell layers is fundamental for device optimization, however no detailed analysis of the influence of growth conditions has been published to date.

We study homogeneous arrays of gallium nitride core-shell microrods with height and diameter in the micrometer range and grown in a two-step selective area MOVPE process. Changes in shell shape and homogeneity effected by deliberately altered shell growth conditions were accurately assessed by digital analysis of high-resolution scanning electron microscope images.

Most notably, two temperature regimes could be established, which show a significantly different behavior with regard to material distribution. Above $900{ }^{\circ} \mathrm{C}$ of wafer carrier temperature, the shell thickness along the growth axis of the rods was very homogeneous, however variations between vicinal rods increase. In contrast, below $830^{\circ} \mathrm{C}$ the shell thickness is higher close to the microrod tip than at the base of the rods, while the lateral homogeneity between neighboring microrods is very uniform. This temperature effect could be either amplified or attenuated by changing the remaining growth parameters such as reactor pressure, structure distance, gallium precursor, carrier gas composition and dopant materials. Possible reasons for these findings are discussed with respect to GaN decomposition as well as the surface and gas phase diffusion of growth species, leading to an improved control of the functional layers in nextgeneration 3D V-III devices.
\end{abstract}

\section{Introduction}

In recent years, many publications have been released concerning the growth of three-dimensional structures. The applications are manifold - future three-dimensional LED structures [1,2] are envisioned as well as three-dimensional (3D) GaN transistors [3], sensors [4] and solar-cells [5]. The structures take the form of either pyramids [6], nano- or microrods as well as GaN fins [7], but also hybrid forms $[8,9]$ have been demonstrated.

In the majority of structures grown by metal organic vapor phase epitaxy (MOVPE), an epitaxial core-shell geometry is employed for gaining an advantage over planar state-of-the art devices. In core-shell configuration, controlling the shape of the shell is fundamental for device optimization, for example in the
InGaN quantum well of 3D LEDs, where growth rate inhomogeneity leads to wavelength gradients along the structures [10-12] due to the indium incorporation dependency on the local growth rate [13] and due to confinement effects in narrow quantum wells. In this publication, we show the result of extensive investigations of the influence of the major MOVPE growth parameters on the morphology of the microrod (MR) shell.

First the growth method for the core-shell MRs is given, then the analytic procedure for the shell layers will be described. The main part shows the specific effects of the investigated growth parameters in subsequent individual sections. Finally, the findings are summarized and a physical hypothesis is established.

\section{Experimental}

The growth templates for the microrods consist of n-doped GaN on $4^{\prime \prime}$ sapphire wafers on which a $100 \mathrm{~nm}$ thick $\mathrm{SiO}_{\mathrm{x}}$ mask layer was deposited by PECVD. The mask layer was uniformly structured 


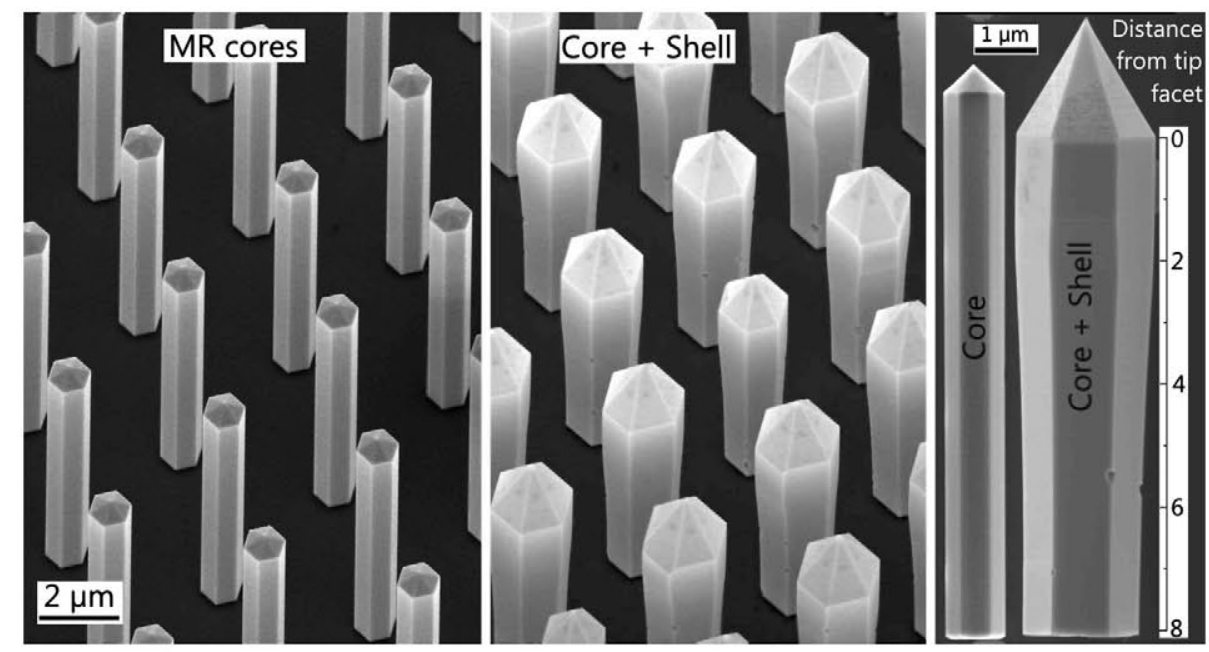

Fig. 1. Array of microrod cores (left) and core-shell structures (center). Right: Collage of lateral images of microrod core and core-shell structure.

with a hexagonal lattice of round $800 \mathrm{~nm}$ diameter openings with a $4 \mu \mathrm{m}$ pitch using nanoimprint and dry etching. On these templates, the core-shell structures were grown in one step in a commercial large-volume MOVPE reactor equipped with in-situ temperature monitoring equipment. The MR cores were grown under conditions similar to those previously published [14], under very low V/III ratio and with a high silane flux. The cores form perfectly regular hexagonal columns, with high homogeneity in lateral direction and without any noticeable tapering along their height (Fig. 1, left). The pitch and large-axis diameter of the MR cores are $4 \mu \mathrm{m}$ and $1050 \mathrm{~nm}$, respectively, the height is approximately $10 \mu \mathrm{m}$. After core growth, conditions were switched to be beneficial for shell layer growth, namely high V/III ratio $(\approx 20,000)$. An AlGaN layer with a thickness of a few nm was deposited to promote smooth shell layer growth on the silicon-rich surface of the highly doped cores [14], followed by a shell of $\approx 400 \mathrm{~nm}$ thickness (Fig. 1, center). Due to the lower growth rate of the semipolar $\{1-101\}$ tip facets of the shell, the resulting m-plane height decreases with the shell layer thickness in accordance to the Wulff-Curie law [15] (Fig. 1, right).

\section{Method of analysis}

Unless specified otherwise, the homogeneity of the shell layers along the growth direction was analyzed by the following procedure: Lateral images of several MRs were taken with an SEM at high resolution and the MR shape was digitized. The circumdiameter of the Core-Shell rod $\left(\mathrm{r}_{\mathrm{CS}}\right)$ was evaluated along its height as the respective distance between the nonpolar sidewalls. Subtracting the previously measured circumdiameter of the MR core $\left(2 r_{\text {Core }}\right)$ and dividing by a factor of two yielded the layer thickness $d$ on an edge of the MR along its entire length. However, the increase of the layer thickness on the side facet of the MR for a given amount of deposited material is proportional to the square of the total rod diameter.

Since the amount of deposited shell material needed to increase the layer thickness increases with the square of the rod radius, the layer thickness is not a good, linear indicator for the material deposition. We therefore calculate the cross-sectional area of the shell $\left(A_{\text {shell }}\right)$ at each measured height, which scales linearly with the amount of deposited material.

$$
\begin{aligned}
A_{\text {shell }} & =A_{C S}-A_{\text {Core }}=\frac{3}{2} \sqrt{3}\left(r_{\text {CS }}^{2}-r_{\text {Core }}^{2}\right)=\frac{3}{2} \sqrt{3}\left(\left(r_{\text {core }}+d\right)^{2}-r_{\text {Core }}^{2}\right) \\
& =\frac{3}{2} \sqrt{3}\left(d^{2}+2 r_{\text {core }} d\right)
\end{aligned}
$$

As shown in Fig. 2, $A_{C S}$ and $A_{\text {Core }}$ denote the cross-sectional area of the core-shell microrod and of the core, respectively, $r_{C S}$ and $r_{\text {Core }}$ are the corresponding circumradii. For simplifying the formula it was used that $A_{C S}=A_{\text {Core }}+A_{\text {Shell }}$. Integrating over the crosssectional area of the shell along the length of the rod yields the total volume of the shell deposited on the core. It is important to note that the cross-sectional area will be shown versus the distance from the tip facet, as shown in the scale in Fig. 1, right.

To account for different growth rates and precursor gases used in this study, $A_{\text {shell }}$ is normalized with regard to the total amount of gallium precursor used, resulting in units given as $\mathrm{nm}^{2} / \mathrm{nmol}$. The pointwise averaged values for all investigated rods from one sample were then compared to those of samples obtained under different growth conditions.

For the analysis of the homogeneity of the MR shape cross section, high resolution SEM images were taken in top view geometry. These images contain several hundreds of individual rods, whose individual cross section areas and diameters were analyzed. To assess the homogeneity of the cross section area - corresponding to the uniformity of material distribution between individual MRs - the standard deviation of the cross section area $\sigma_{A}$ is taken as the figure of merit. A large value of the standard deviation indicates large fluctuations in the material distribution and the rods are differing significantly in shell volume. Furthermore, the homogeneity of the core-shell rod shape was analyzed. While the MR

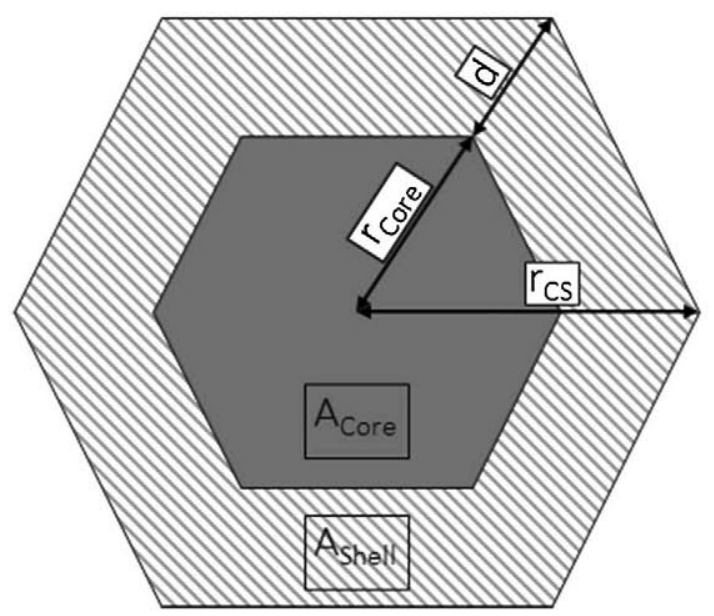

Fig. 2. Cross-sectional areas and diameters of a core-shell microrod. 
cores have close to perfectly uniform hexagonal cross sections, the shell layer thickness can fluctuate strongly on adjacent facets of a core-shell MR, leading to a distortion of the hexagonal rod shape. To assess this behavior, we measured the smallest and largest Feret diameter (or caliper diameter) of the rods in the SEM images. The Feret diameter extrema correspond to the diameter between opposite sides and corners of the hexagon, respectively. For a perfectly regular hexagon, the ratio between the smallest and largest Feret diameter is $\sqrt{3} / 2$; if the hexagon is distorted, the ratio must always be smaller. Thus, by calculating the difference of the mean ratio from $\sqrt{3} / 2$, we obtain a figure of merit for the homogeneity of the cross-sectional shape of MRs, which will be called $\Delta_{\mathrm{Hex}}$.

$\Delta_{\text {Hex }}=\frac{\sqrt{3}}{2}-\frac{1}{n} \sum_{i=1}^{n} \frac{D_{i}^{\min \text { Feret }}}{D_{i}^{\max } \text { Feret }}$

The larger this figure, the more irregular is the shape of the rods on that sample. The number $\mathrm{n}$ in the above formula denotes the number of measured rods.

\section{Study of shell growth conditions}

In the following section, the most important parameters for the GaN growth of three dimensional structures are studied. The primary growth parameters consisting of the growth temperature, pressure and carrier gas composition were varied. Additionally, the difference between the use of Trimethylgallium (TMGa) and Triethylgallium (TEGa) were evaluated and the effects of doping with silane $\left(\mathrm{SiH}_{4}\right)$ for $\mathrm{n}$-doping and $\mathrm{Bis}$ (cyclopentadienyl)magnesium $\left(\mathrm{Cp}_{2} \mathrm{Mg}\right)$ for $\mathrm{p}$-doping on the shape of the layers was assessed. Lastly the effects of variations in the array spacing were examined, showing the dependency of the shell shape on this geometrical factor.

\subsection{Influence of shell growth temperature}

For this study, the microrod GaN shell was grown at several temperatures and its thickness analyzed with the method detailed above. For a range of temperatures, the variation of the crosssectional shell area along the vertical axis of the microrod is shown in Fig. 3a. We consider the point closest to the tip inclined facet to be at $0 \mu \mathrm{m}$ and measure towards the MR base, which is situated at $8 \mu \mathrm{m}$.

At growth temperatures of $880^{\circ} \mathrm{C}$ and above (yellow, red and purple curve), the $\mathrm{m}$-plane side facets of the structures are almost completely vertical, with only a minor amount of tapering detectable - the structures are slightly wider at their foot point as com-

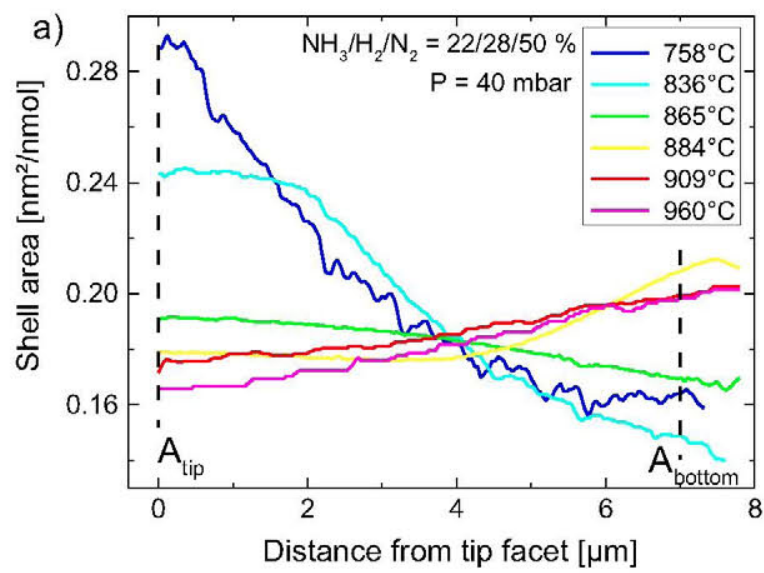

pared to the diameter at the tip. For temperatures of $830^{\circ} \mathrm{C}$ and below (cyan and blue curve), the thickness gradient is inverted the structures are distinctly narrower at the foot point than at the upper part of the rod. In between those temperatures exists a transition regime with intermediate shape (green curve).

For analysis of the nature of this transition, we define two measurement points: $A_{\text {up }}$ is the shell area at the top of the rods, close to the transition to the semipolar facets. $A_{\text {bottom }}$ is the shell area very close to the mask layer, at a distance of $7 \mu \mathrm{m}$ from $A_{u p}$. The position of these points is specified by two dashed thin black lines in Fig. 3a.

The ratio of the shell area close to the tip facet $\left(A_{u p}\right)$ and one micrometer above the mask $\left(A_{\text {bottom }}\right)$ is shown as the solid square points in Fig. 3b for all four microrods measured per sample. The points have been fitted with a logistic function of the form

$\frac{A_{\text {bottom }}}{A_{\text {tip }}}=A_{1}+\frac{A_{1}-A_{2}}{1+\left(\frac{T}{T_{0}}\right)^{p}}$

Here, $\mathrm{A} 1=0.589 \pm 0.008$ and $\mathrm{A} 2=1.174 \pm 0.008$ denote the constant values at start and finish of the transition, respectively. $\mathrm{T}_{0}=864.7 \pm 0.4{ }^{\circ} \mathrm{C}$ specifies the central point of the transition and $\mathrm{p}=150 \pm 30$ its steepness.

The function fits perfectly to the steep transition between two regimes and indicates a start of the shape transition at $830^{\circ} \mathrm{C}$ as well as its completion at $900^{\circ} \mathrm{C}$. For the rest of this article, we will be referring to the two ranges with constant tapering as the high and low temperature regime, respectively.

Integrating over the area curves of Fig. 3a allows to determine the total amount of material deposited during shell growth. The shell volume of the analyzed rods is shown in the open circles in Fig. 3b). A behavior inverse to the evolution of the tapering can be noticed and a fit with the logistic function was applied using the same steepness $\mathrm{p}$ as for the shell area ratio resulting in upper and lower bounds of $\mathrm{A} 1=22.89 \pm 0.10 \mu \mathrm{m}^{3}$ and $\mathrm{A} 2=21.84 \pm$ $0.08 \mu \mathrm{m}^{3}$, respectively, and the central temperature of $\mathrm{T}_{0}=862 \pm 3^{\circ} \mathrm{C}$. While the uncertainty of the fit is much higher due to the relatively small change in total shell volume and the limited accuracy of the measurement, the reasonable agreement of the fit to the data indicates a link between the deposited volume and the homogeneity. A hypothesis concerning the nature of this phenomenon will be discussed in Section 5.

\subsection{Influence of the growth pressure}

In the previous paragraph, two temperature regimes have been established, which show a distinctly different material distribution

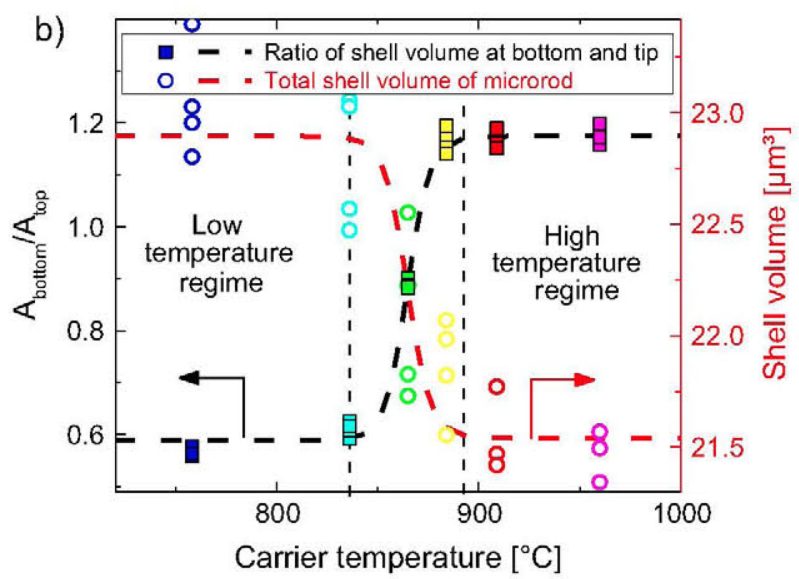

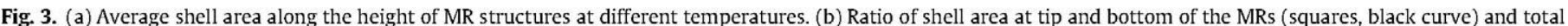

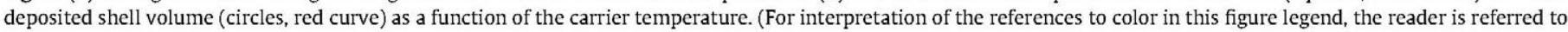
the web version of this article.) 

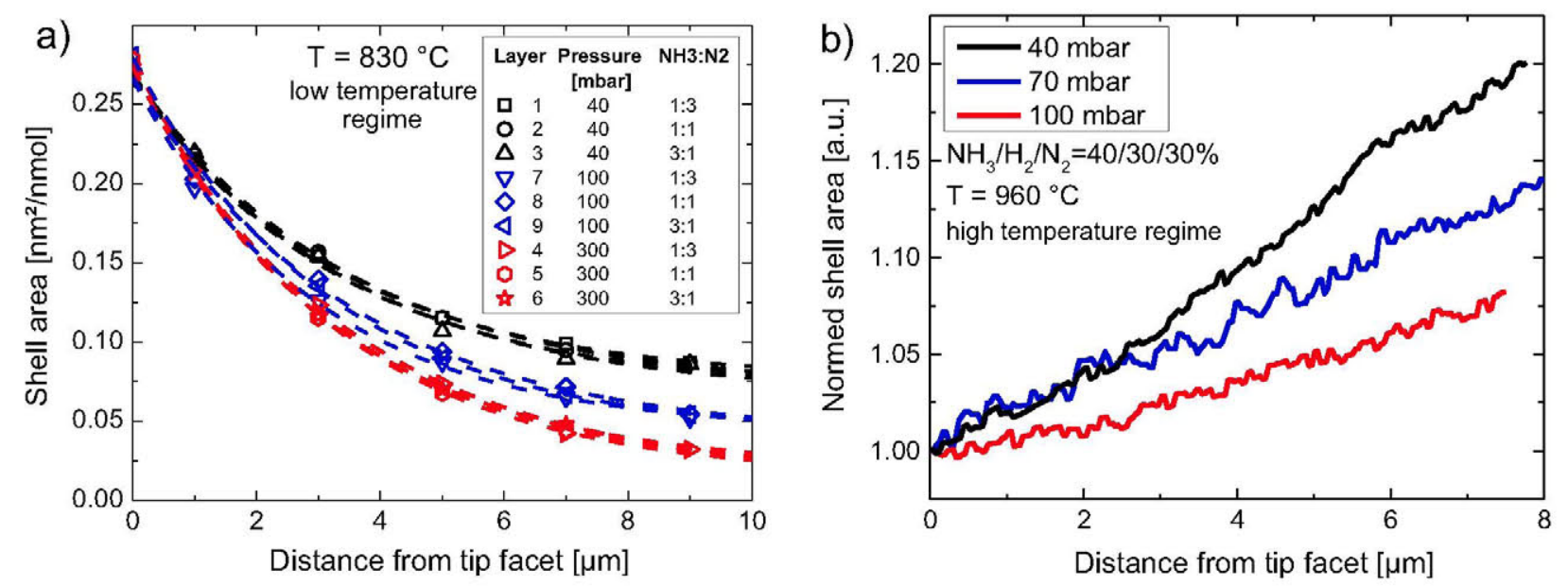

Fig. 4. (a) Shell area over height at different growth pressure in the low temperature regime. (b) Shell layer thickness versus height in the high temperature regime.
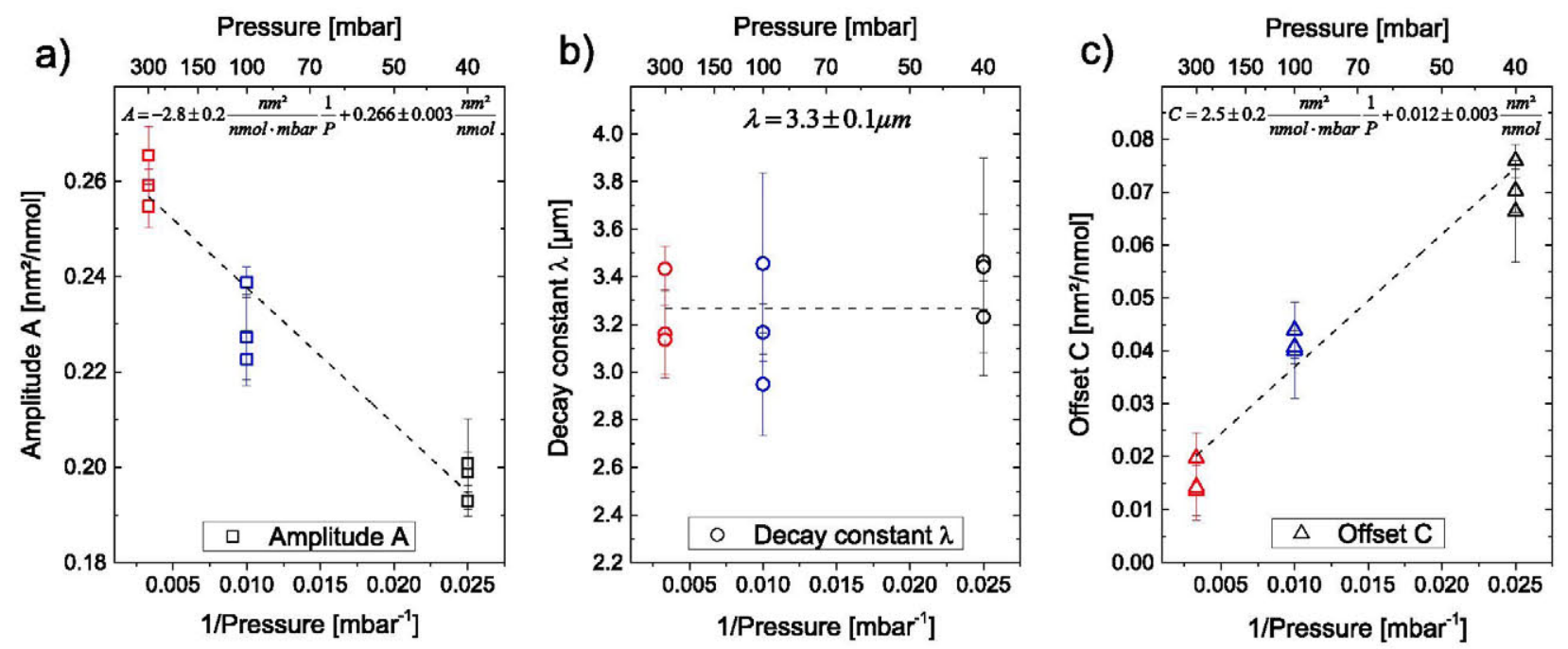

Fig. 5. (a) Amplitude, (b) decay constant and (c) offset from the exponential fits of Fig. 4a, with linear fits to the data.

along the microrods. In this paragraph, we report the effects of pressure in the two separate regimes. Additionally, the lateral homogeneity of the microrods with respect to temperature and pressure will be evaluated.

A special test structure was designed, consisting of thin GaN test layers grown on MR cores at $830^{\circ} \mathrm{C}$ and varying pressure, growth rate and $\mathrm{NH}_{3} / \mathrm{N}_{2}$ ratio but without hydrogen, using TEGa as Ga-precursor and with AlGaN interlayers to provide contrast. A TEM lamella was prepared from this sample and the thickness of the individual layers was measured at six points along the MR. The shell area is shown in Fig. 4a, normalized with regard to growth time and Ga-precursor flow. The individual points were phenomenologically fitted with a function of the form

$A_{\text {shell }}=A \exp \left(-\frac{h}{\lambda}\right)+C$

which is an exponential decay with initial Amplitude A and decay constant $\lambda$, and an offset of $C$.

For $\mathrm{A}$ and $\mathrm{C}$ a direct relation to the inverse pressure is found. The fitting parameters are shown in Fig. 5 along with a linear fit over the inverse pressure. The modulation ratio of amplitude to offset $\mathrm{A} / \mathrm{C}$ (Fig. 5a and $\mathrm{c}$ ) decreases with decreasing pressure and by decreasing the pressure the offset will increase. These factors lead to an increasing homogeneity of the material distribution along the microrods with lower pressure. Interestingly, the decay constant $\lambda$ (Fig. 5b) does not change decisively within the precision of the exponential fits over the measured pressure range.

The results indicate that lower pressure helps to obtain a lower shell area gradient along the MR m-plane in the low temperature regime. Growth rate variations between 10 and $100 \mathrm{~nm} / \mathrm{h}$ resulted in no measurable difference regarding the anti-tapering and neither did the variation in V/III ratio.

For the high temperature regime, three growth runs were conducted with shell growth at $960^{\circ} \mathrm{C}$ and 40,70 and $100 \mathrm{mbar}$, respectively. They were evaluated with the standard analysis method detailed in the third section of this publication. The resulting diameter curves are shown in Fig. 4b, normalized to the thickness at the tip due to the lateral inhomogeneity in the high temperature regime. While only a minor effect to the gradient is observed, a clear trend develops, indicating that the MR shell grown at high pressure is slightly more homogeneous along the m-plane facet of the MR than the shell grown at lower temperature.

After investigating the homogeneity of the layers along the microrods, the dependence of the lateral homogeneity on temperature and pressure will be surveyed. Although the initial array of hexagonal microrod cores are very uniform, the growth of the shell 

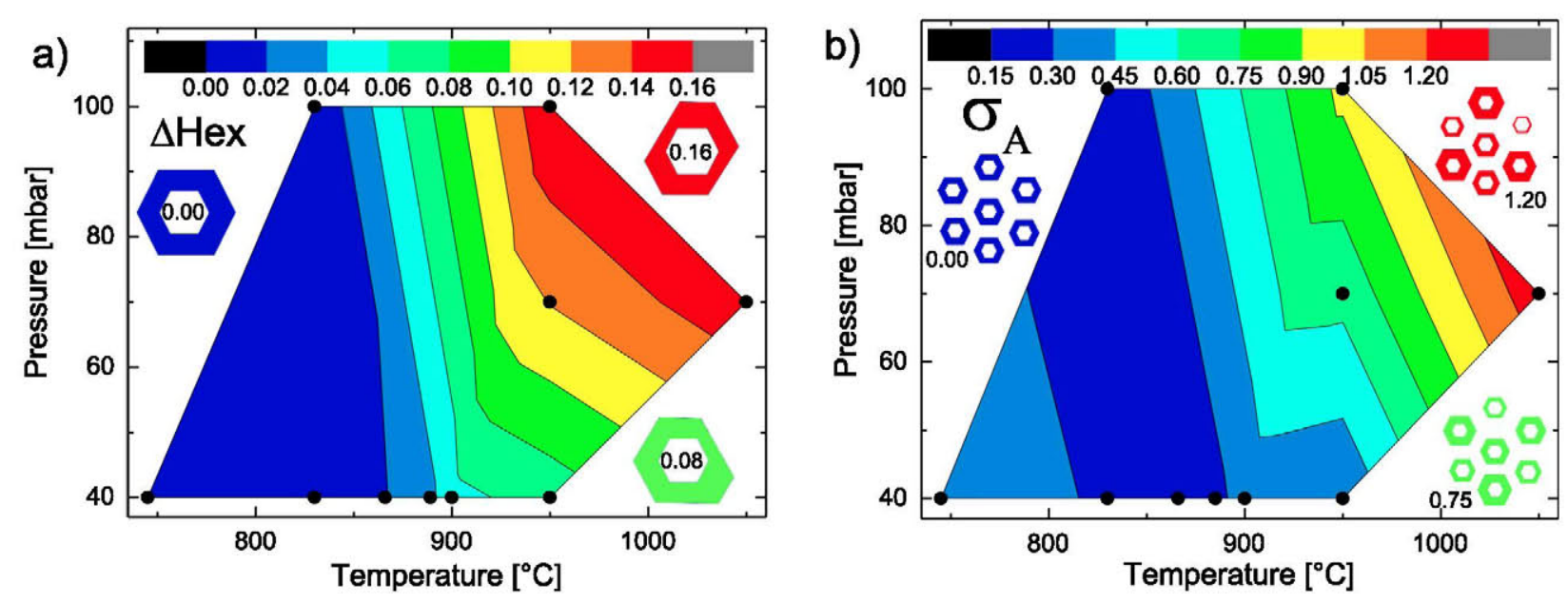

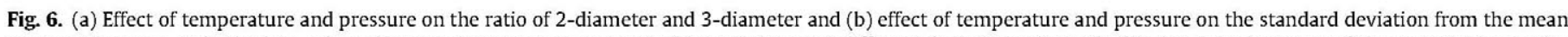

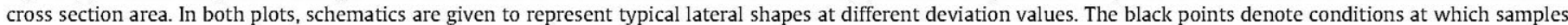
were grown.

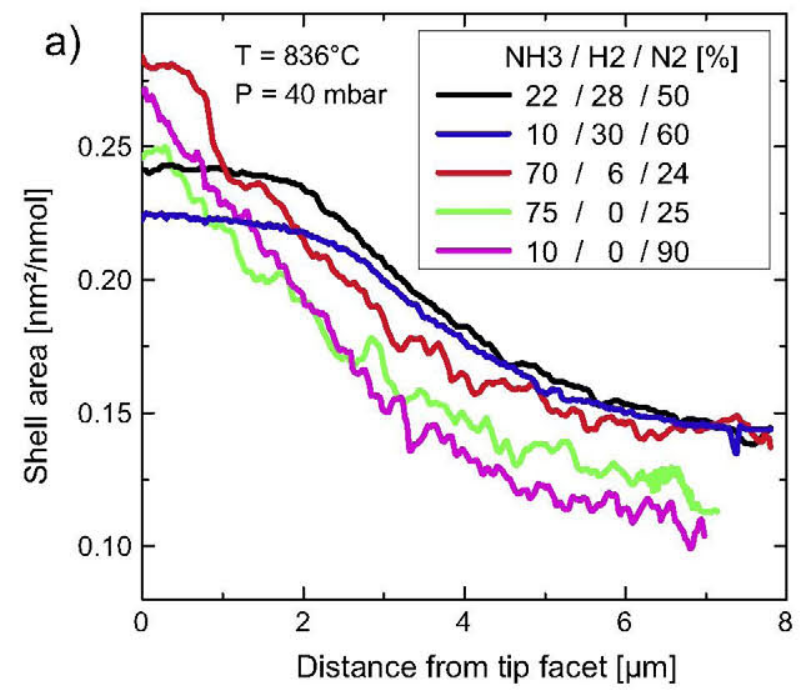

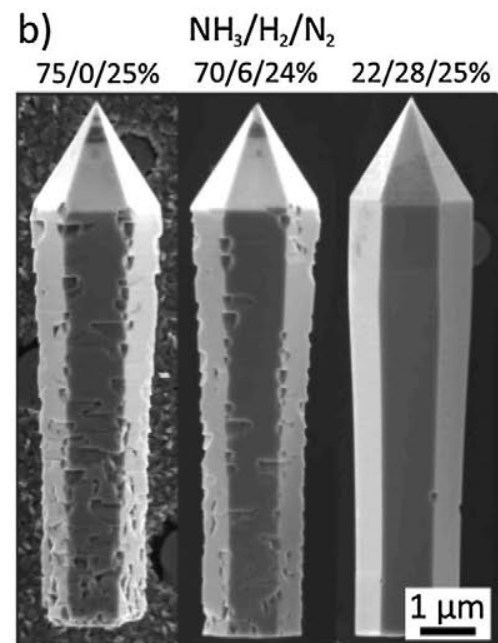

(I)

(II)

(III)

Fig. 7. (a) Shell layer area of samples grown under different carrier gas mixture. (b) Comparison between individual MRs and influence of hydrogen flow.

can lead to the introduction of significant irregularity in lateral direction. The figures of merit $\Delta_{\mathrm{Hex}}$ and $\sigma_{A}$, whose derivation is described in paragraph 3 , are plotted depending on the growth temperature and pressure in Fig. 6a and b.

One can observe that the temperature plays the dominating role in the lateral homogeneity of the rods: while at temperatures up to $850^{\circ} \mathrm{C}$ the array is very uniform in area and shape of the rods as indicated by the solid blue color of the section, at higher temperature the inhomogeneity starts to increase rapidly. The similarity of the crossover point in the longitudinal homogeneity of the rod shell and the lateral homogeneity of the cross section is notable which will be discussed in Section 5 .

In the low temperature regime, there is no effect of the pressure on the lateral homogeneity. However, low pressure can decrease the cross-sectional inhomogeneity produced by high-temperature growth - a shell layer deposited at $950^{\circ} \mathrm{C}$ and 40 mbar is much more homogeneous than one deposited at 100 mbar.

\subsection{Influence of the carrier gas mixture}

Besides the temperature and pressure, another significant parameter in MOVPE growth is the composition of the carrier gas, meaning the relative proportion of ammonia, hydrogen and nitrogen which constitute the majority of gas flow through the reactor. To investigate this parameter, microrod shells were grown under a range of different gas mixtures and their cross-sectional area along the height is shown in Fig. 7a. A growth temperature of $835^{\circ} \mathrm{C}$, close to the transition region was used to be as sensitive as possible to changes in the shell area gradient.

No influence of the $\mathrm{V} / \mathrm{III}$ ratio or the $\mathrm{NH}_{3}$ content of the gas mixture could be detected as indicated by the similarity of the green and magenta area curve in Fig. 7a and as already suggested by the individual curves in Fig. $4 \mathrm{a}$. However, the hydrogen content of the gas mixture has a noticeable effect. For growth at low temperature without hydrogen addition, parasitic growth of GaN crystals on the $\mathrm{SiO}_{\mathrm{x}}$ mask could be detected. This is visible in the background of the MR in Fig. 7(b, I). The addition of a small amount of hydrogen increased the selectivity of the $\mathrm{SiO}_{\mathrm{x}}$ mask so that no parasitic growth on the mask occurred (Fig. 7b II). Nevertheless, no major influence on the tapering could be detected ( red $^{1}$ curve), the corresponding area curve is parallel to those for growth without

\footnotetext{
${ }^{1}$ For interpretation of color in Figs. 7, 9 and 10 , the reader is referred to the web version of this article.
} 


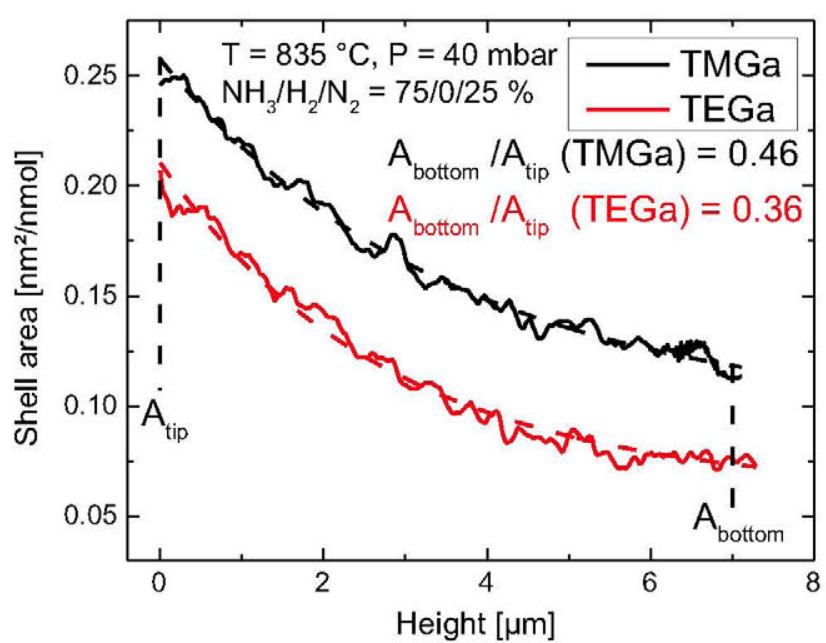

Fig. 8. Shell area profile for microrod shell grown with TMGa (black) and TEGa (red) and ratio of bottom to tip shell area. (For interpretation of the references to color in this figure legend, the reader is referred to the web version of this article.)

hydrogen. For higher hydrogen content, the anti-tapering decreased slightly, with a vertical section forming on the m-plane close to the MR tip and the GaN layer got significantly smoother (Fig. 7b III).

The gas mixture used during growth thus has a slight influence on the vertical distribution of the shell material, especially close to the transition between the two temperature regimes. The lateral homogeneity of all samples is close to perfect at this low temperature and pressure and no significant difference between the individual runs was detected. However, the material quality of low temperature GaN shells can be improved significantly by the addition of hydrogen to the carrier gas, as can be inferred from the substantial smoother surface of the nonpolar GaN facet for an increased $\mathrm{H}_{2}$ ratio.

\subsection{Influence of the gallium precursor gasses}

Two precursor gasses are routinely used in GaN growth: Trimethylgallium (TMGa, $\mathrm{C}_{3} \mathrm{H}_{9} \mathrm{Ga}$ ) and Triethylgallium (TEGa, $\mathrm{C}_{6} \mathrm{H}_{15} \mathrm{Ga}$ ). The main difference is the vapor pressure, but also the dissociation temperature and decomposition pathway during the growth process is different [16]. For most of the growth runs in this experimental series, TMGa was used as the gallium precursor. To investigate the effect of using TEGa in the low temperature regime, two growth runs were conducted. $\mathrm{No}_{2}$ was used in the carrier gas mixture to obtain conditions similar to InGaN quantum well growth. The shell area curves are shown in Fig. 8. The growth rate is lower for TEGa-based growth than for TMGa-based growth even if the molar flow of the precursor gasses is equal, as can be deduced from the vertical spacing between the normed shell area curves. By comparing the ratio of the bottom shell area $A_{\text {bottom }}$ to the shell area at the tip $A_{\text {tip }}$, we can deduce that the relative tapering for TEGa-based GaN growth is higher than for TMGa-based GaN growth. A fit of the area curves with the adjusted exponential function of paragraph 4 shows that the decay constant for TMGa $(\lambda=3.55)$ is slightly higher than for TEGa $(\lambda=2.80)$, indicating a more homogeneous material distribution. No difference was observed for the lateral homogeneity, at this low temperature and pressure both samples show core-shell rods with close to perfectly homogeneous hexagonal cross-sections.

\subsection{Effect of doping on material distribution in high temperature regime}

Additional investigations were made on the influence of the standard precursor materials for $\mathrm{n}$ - and $\mathrm{p}$-type doping, Silane $\left(\mathrm{SiH}_{4}\right)$ and $\mathrm{Bis}\left(\mathrm{cyclopentadienyl)magnesium}\left(\mathrm{Cp}_{2} \mathrm{Mg}\right)\right.$. No major change in the material distribution was observed under the addition of a moderate amount of silane which would result in a silicon concentration of about $5 \cdot 10^{18} \mathrm{~cm}^{-3}$ for a planar c-plane GaN layer (Fig. 9, black curve).

Contrarily, the addition of the Mg-precursor leads to significantly enhanced tapering, especially at low pressure (Fig. 9, blue curve). This effect is reduced by growing the p-doped GaN shell at elevated pressure of $100 \mathrm{mbar}$ or higher (Fig. 9, red curve). Under these conditions, the tapering is reduced close to that of an undoped GaN shell at 40 mbar. The applied doping concentration would result in a magnesium concentration of $2 \cdot 10^{19} \mathrm{~cm}^{-3}$ on a planar c-plane GaN layer.

For all examined microrods with p-doped shell layers, a contrasted area with triangular shape was visible on the tip facets under the chosen SEM imaging parameters. A reduction in size for reduced acceleration voltages hints at a very low thickness of the p-doped GaN shell at this point of the rod, which is in the range of the penetration depth of the electron beam into the GaN. Addi-
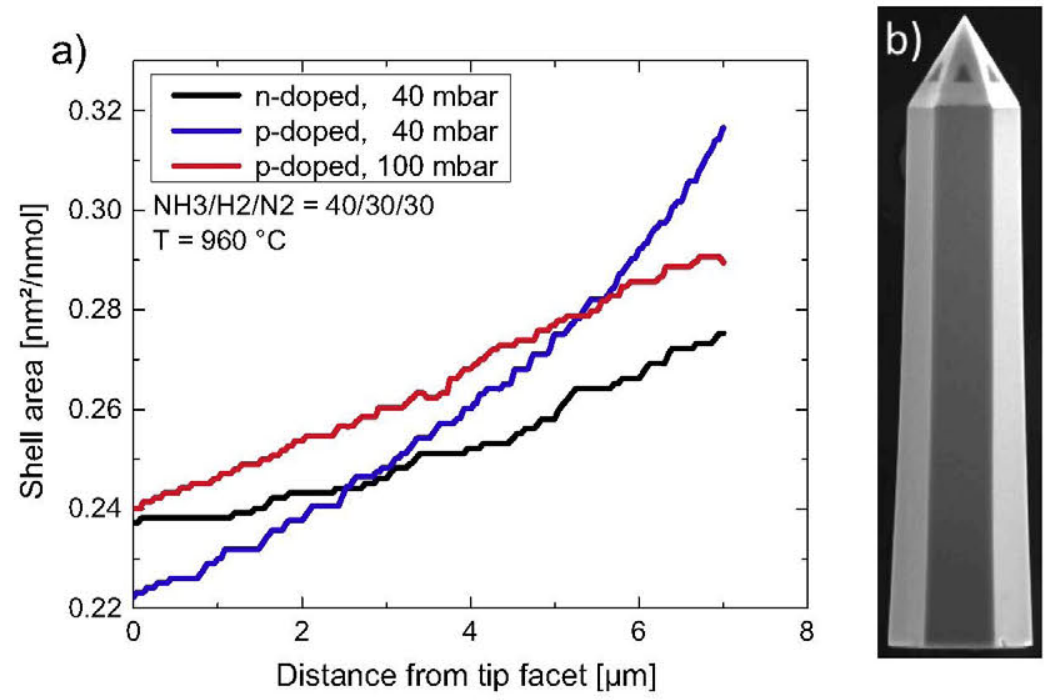

Fig. 9. (a) Shell area for shells with n- and p-doping. (b) SEM image of core-shell structure with p-doping at low pressure. 




Fig. 10. Box-plot of the lateral inhomogeneity parameter $\Delta \mathrm{Hex}$ for differently doped shells with data points from several hundred microrods of each sample.

tional investigations have to be conducted to clarify this discovery and its impact for core-shell microrod based devices.

The lateral homogeneity of the doped rods is shown in a box plot in Fig. 10. The addition of $\mathrm{SiH}_{4}$ and $\mathrm{Cp}_{2} \mathrm{Mg}$ lowers the figure of merit $\Delta \mathrm{Hex}$, as can be seen when comparing the blue and purple boxes to the black box representing the undoped reference sample. This homogenizing effect seems to be slightly more pronounced for the p-doped sample. While for n-doping the established trend to inhomogeneity can be observed for higher pressure growth (compare Fig. 4), this effect seems to be suppressed in the p-doped samples. The lateral homogeneity did not increase considerably under increased growth pressure, as can be seen by comparing the magenta and green boxes. The $\sigma_{A}$ shows the same dependency.

\subsection{Variation of the distance between structures}

Another major influence on the tapering comes from the spacing between two adjacent microrod structures, however, this only is influential in the low temperature regime. In the high temperature region, even very dense arrays of microrods can be covered with a conformal shell until almost complete coalescence, as shown in Fig. 11a. However in the low temperature regime, the amount of tapering is significantly dependent on the space between the microrod side walls. In Fig. $11 \mathrm{~b}$, the shell area gradient for three different microrod side wall distances is plotted for a growth temperature of $830^{\circ} \mathrm{C}$ and without hydrogen addition. The clear influence of closely standing microrods is obvious from the sharp decline of the shell area in the first two micrometers for the densest array. The curves were fitted with exponential decays of the form $A=\exp (-h / \lambda)+C$ as in Section 4.2. The decay constant $\lambda$ and offset $C$ are plotted versus the side wall distance in Fig. 11c and were fitted with linear functions of the form $y=a \cdot x$. No offset was introduced since it makes sense from a physical point of view for offset and decay constant going to zero for a vanishing distance between the microrods. For the amplitude A of the exponential term, a linear relationship to the distance could be observed, too, although with a y-axis offset.

These results indicate a severe limitation of the material transport onto the side walls of closely arranged MRs in the low temperature regime.

\section{Discussion}

We have performed a thorough analysis of the influence of different shell growth conditions on the lateral and vertical homogeneity. The growth temperature was identified as the most crucial parameter and two different temperature regimes were established.

At temperatures of $830^{\circ} \mathrm{C}$ and below the shell shows a distinctive anti-tapering and more shell material is deposited at the top of the MR than at the bottom. Growing at a relative low reactor pressure of 40 mbar increases the vertical homogeneity and more material reaches the lower part of the MR. Using TEGa precursor instead of TMGa increases the thickness gradient slightly. Adding hydrogen to the carrier gas mixture leads to better material distribution and increased crystal quality as suggested by the improved surface smoothness. The lateral homogeneity is excellent in the low temperature regime and the MR cross sections form almost perfectly regular hexagons over the investigated pressure range.

At temperatures of $880^{\circ} \mathrm{C}$ and higher, the shell grows quite uniformly along the height of the rods with a small tapering due to a material surplus at the bottom part of the rod, however the lateral homogeneity decreases strongly with temperature. Growth at lower pressure leads to slightly higher tapering, however the lateral homogeneity increases notably. While n-type doping of the shell has no major effect on the material distribution, doping the
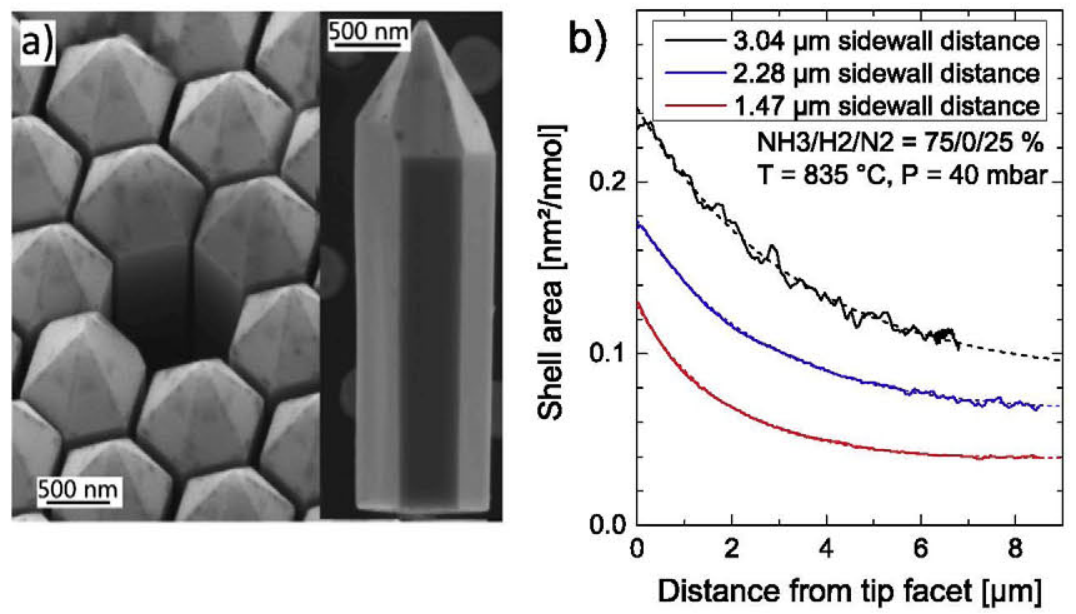

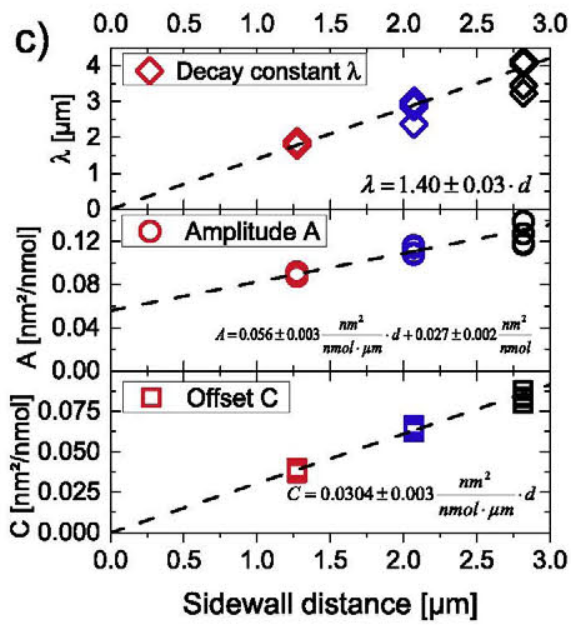

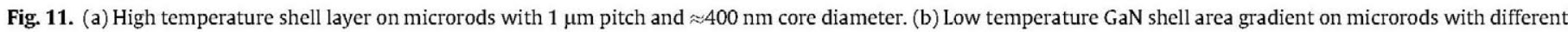
side wall distance. (c) Linear fit to decay constants and offsets from the fits in figure (b). 
shell layer with $\mathrm{Cp}_{2} \mathrm{Mg}$ for $\mathrm{p}$-conductivity distinctly increases the tapering and a much higher amount of material is deposited close to the foot point of the MR. This behavior can be reduced by increasing the growth pressure for the p-doped shell layer and at a pressure of 100 mbar the tapering of the p-doped GaN shell is reduced close to the undoped reference.

To understand these results, some considerations about the growth environment have to be taken into account. For the reactor type and growth parameters used in this study, the thickness of the thermal and momentum boundary layer above the wafer is between one and two centimeters, approximately three orders of magnitude larger than the height of the MRs in the array. We can assume that the temperature difference between the rod tip and the substrate is very low $\left(\approx 0.1^{\circ} \mathrm{C}\right)$, and that no direct material transport by gas flow is present in or directly above the MR array. Therefore, the only transport mechanism into the MR array and onto the MR side walls can be by gas diffusion. As pointed out by Lymperakis and Neugebauer [17], the m-plane side walls show a large anisotropy of the gallium diffusion barrier. Surface diffusion in axial c-direction $\left(\Delta E_{\| 0001]}^{m-p l a n e}=0.93 \mathrm{eV}\right)$ is significantly restricted by the more than four times stronger barrier than in the lateral a-direction $\left(\Delta E_{\perp[0001]}^{m-p l a n e}=0.21 \mathrm{eV}\right)$. For comparison, the surface diffusion barrier on c-plane (0001) GaN surfaces is isotropic with a value of $\approx 0.4 \mathrm{eV}$ [17]. This results in a limited diffusion-based transport of gallium along the growth axis of the MRs.

A thorough physical treatment of the problem at hand is difficult since the mean free path in the gas mixture is about equal to the distance between two MRs. The system is in the transition regime between classical continuum fluid dynamics and free molecular flow, which complicates analytical description. Instead, we offer some thoughts about the possible reasons leading to the observed effects for shell growth.

A first hypothesis would be that a lack of available nitrogen is the cause of the layer thickness variation. While the decomposition of $\mathrm{NH}_{3}$ is significantly changing in the investigated temperature range [18], this theory is unlikely. In the case of nitrogen limitation, the variation of the V/III ratio and the subsequent change in ammonia partial pressure should result in a change of the shell area gradient which has not been observed.

Secondly, the capture of material through parasitic growth on the mask layer at low temperatures could also be ruled out. While parasitic growth on the mask was identified at low temperature along with the layer thickness gradient, the addition of a minor amount of hydrogen to the carrier gas mixture completely removed the parasitic growth without changing the shell area gradient.

A third theory based on an additional material transport channel over the $\mathrm{SiO}_{\mathrm{x}}$ mask layer, which only comes into play at a certain activation temperature can also be dismissed. The analysis of the shell volume curves in Fig. 3b shows that the increased homogeneity at high temperatures comes from material redistribution and not from an additional material source.

A more plausible explanation can be based on the observation that the material distribution in vertical and lateral direction changes significantly at a temperature around $830^{\circ} \mathrm{C}$. This is the temperature at which the GaN surface starts to decompose measurably, and species are desorbing with exponential dependency on the temperature $[19,20]$, although the melting point of GaN is about $1500^{\circ} \mathrm{C}$ higher [21]. Such a decomposition process leads to the recapture of growth species into the gas phase and their redeposition at another position. This could explain the rapid change in the layer thickness above $830^{\circ} \mathrm{C}$. Further arguments for this thesis are given by the observed smoothing of the exponential profile and the slight reduction in the deposition rate, which is expected for higher temperature due to the escape of a portion of the desorbed material to the reactor outlet. The decrease of the lateral homogeneity with increasing temperature also indicates an increased material diffusion to adjacent facets and neighboring MRs, the negative influence of pressure on the lateral homogeneity at high temperatures follows the results of Koleske et al. for increased GaN desorption at higher pressure [19].

We see that at low temperatures the pressure has a significant effect on the vertical homogeneity. While the decay constant is not changed detectably, the constant offset and amplitude are reduced and increased with higher pressure, respectively. The GaN decomposition noted above plays no role at this low temperature. We suspect that a gas phase diffusion related transport mechanism is responsible for the improved material distribution, but further research has to be done to further clarify the exact process.

Another investigated growth parameter was the GaN precursor and carrier gas used for the growth. The difference in the shell area profile between TMGa and TEGa can be explained by the different molecular sizes of the precursor gasses. The precise decomposition path of the gases and whether this occurs in the gas phase or on surfaces is still under discussion. The observed differences in the decay constant hints towards a lower diffusion coefficient in the gas phase, possibly caused by a higher molecular diameter of the TEGa decomposition product compared to that of TMGa.

The addition of hydrogen to the carrier gas increases the homogeneity of the shell layer slightly and removes parasitic GaN growth on the $\mathrm{SiO}_{\mathrm{x}}$ mask layer. The largest effect can be seen close to the transition regime at a temperature of $830^{\circ} \mathrm{C}$. The investigation of the integrated shell area reveals that the improved homogeneity is achieved by a redistribution of the material from the area close to the tip towards the MR base. While $\mathrm{H}_{2}$-promoted etching only plays a major role at temperatures above $1000^{\circ} \mathrm{C}$, hydrogen is believed to enhance the desorption of adsorbed $\mathrm{Ga}$ atoms from the surface [22] even at low temperature.

Not many publications deal with the change of material distribution seen for doped shells. While no publications state a change in GaN morphology for moderate silicon doping, effects for $\mathrm{p}$ doped GaN have been reported. Beaumont et al. report the suppression of r-plane pyramidal growth and the development of homogeneous c-plane platelets for the growth of p-doped GaN on a patterned $\mathrm{SiN} / \mathrm{GaN} /$ sapphire template [23]. A reduction in $\mathrm{V}$ Pit density in p-doped GaN layers is reported by Chen et al. [24], which is also attributed to the increased lateral growth on $\mathrm{p}$ doped GaN. A smoothing effect of magnesium has also been noticed in the MBE growth of $\mathrm{GaN}$ [25] and was attributed to a surfactant effect due to the lowering of surface diffusion barriers by an electron counting mechanism. It is plausible that the effects described in Section 4.5 are due to an increase of surface diffusion while the diffusion between structures through the gas phase is suppressed, as shown by the lack of decrease in lateral homogeneity for $\mathrm{p}$-doped shells grown at higher pressure.

\section{Conclusion}

As one of the major results of this study, two temperature regimes with distinctly different material distribution along the GaN microrods were established. While at temperatures above $900^{\circ} \mathrm{C}$ the shell layers cover the core very uniformly, for temperatures below $850^{\circ} \mathrm{C}$ significantly more material is deposited close to the tip of the microrods. For most applications a homogeneous shell thickness is beneficial to ensure e.g. homogeneous current transport through the layers along the wire. However an increased shell thickness at the tip of the microrods might be suited for a coalescence of the rods, e.g. to serve as defect-reduced templates [26].

The temperatures usually employed for the growth of InGaN quantum wells with emission wavelengths in the visible range 
are deep in the low temperature regime $\left(<750^{\circ} \mathrm{C}\right)$. Matchingly, distinct anti-tapering of the active layers was detected in many publications on core-shell LED structures [11,27,28]. While a surfactant effect of indium has been proposed for the growth of gallium nitride under certain conditions [29], no significant difference between the thickness distribution of GaN shell layers with and without indium under otherwise identical growth conditions has been observed in our preliminary experiments.

For low-temperature shell layers, growth at low pressure showed the largest impact on the vertical homogeneity. The addition of hydrogen also increased the homogeneity of the layers, as well as the surface smoothness and the selectivity of the $\mathrm{SiO}_{\mathrm{x}}$ mask layer. However this remedy is difficult to apply to the growth of indium containing layers due to its desorption by hydrogen [13].

In general our study shows that growth at low pressure is advantageous for the growth of homogeneous shell layers at all temperatures. The only exception consists of Mg-doped p-GaN shells in the high temperature regime where low growth pressure resulted in strong tapering and the development of a tent-like shape of the MR structure.

While further research has to be done to ascertain the exact cause of the detected phenomena, we are certain that these results are helpful for a multitude of structures involving three dimensional growth of gallium nitride.

\section{Acknowledgements}

The authors thank F. F. Krause and A. Rosenauer for the transmission electron microscope measurements.

\section{References}

[1] M. Mandl, X. Wang, T. Schimpke, C. Kölper, M. Binder, J. Ledig, A. Waag, X. Kong, A. Trampert, F. Bertram, J. Christen, F. Barbagini, E. Calleja, M. Strassburg, Phys. Status Solidi RRL 7 (2013) 800.

[2] S. Li, A. Waag, J. Appl. Phys. 111 (2012) 71101.

[3] F. Yu, D. Rümmler, J. Hartmann, L. Caccamo, T. Schimpke, M. Strassburg, A.-E. Gad, A. Bakin, H.-H. Wehmann, B. Witzigmann, A. Waag, Appl. Phys. Lett. (2016).

[4] J.L. Johnson, Y. Choi, A. Ural, W. Lim, J.S. Wright, B.P. Gila, F. Ren, S.J. Pearton, J. Electr. Mater. 38 (2009) 490

[5] Y. Dong, B. Tian, T.J. Kempa, C.M. Lieber, Nano Lett. 9 (2009) 2183.
[6] T. Wunderer, M. Feneberg, F. Lipski, J. Wang, R.A.R. Leute, S. Schwaiger, K. Thonke, A. Chuvilin, U. Kaiser, S. Metzner, F. Bertram, J. Christen, G.J. Beirne, M. Jetter, P. Michler, L. Schade, C. Vierheilig, U.T. Schwarz, A.D. Dräger, A. Hangleiter, F. Scholz, Phys. Status Solidi B 248 (2011) 549.

[7] J. Hartmann, F. Steib, H. Zhou, J. ledig, S. Fündling, F. Albrecht, T. Schimpke, A Avramescu, T. Varghese, H.-H. Wehmann, M. Strassburg, H.-J. Lugauer, A. Waag, Cryst. Growth Des. (2016).

[8] Y. Tchoe, J. Jo, M. Kim, J. Heo, G. Yoo, C. Sone, G.-C. Yi, Adv. Mater. 26 (2014) 3019 (Deerfield Beach, Fla.).

[9] J.S. Min, J.C. Yun, J.C. Jiho, S.A. Hyung, N.Y. Sam, H.H. Dong, H. Yoon, H.P. Seoung, J. Korean Phys. Soc. 58 (2011) 1351

[10] M. Tchernycheva, V. Neplokh, H. Zhang, P. Lavenus, L. Rigutti, F. Bayle, F.H. Julien, A. Babichev, G. Jacopin, L. Largeau, R. Ciechonski, G. Vescovi, O. Kryliouk, Nanoscale 7 (2015) 11692

[11] C.-H. Liao, C.-G. Tu, W.-M. Chang, C.-Y. Su, P.-Y. Shih, H.-T. Chen, Y.-F. Yao, C Hsieh, H.-S. Chen, C.-H. Lin, C.-K. Yu, Y.-W. Kiang, C.C. Yang, Opt. Express 22 (2014) 17303.

[12] T. Schimpke, M. Mandl, I. Stoll, B. Pohl-Klein, D. Bichler, F. Zwaschka, J. StrubeKnyrim, B. Huckenbeck, B. Max, M. Müller, P. Veit, F. Bertram, J. Christen, J. Hartmann, A. Waag, H.-J. Lugauer, M. Strassburg, Phys. Status Solidi A (2016).

[13] M. Bosi, R. Fornari, J. Cryst. Growth 265 (2004) 434.

[14] J. Hartmann, X. Wang, H. Schuhmann, W. Dziony, L. Caccamo, J. Ledig, M.S Mohajerani, T. Schimpke, M. Bähr, G. Lilienkamp, W. Daum, M. Seibt, M. Straßsburg, H.-H. Wehmann, A. Waag, Phys. Status Solidi A 212 (2015) 2830.

[15] G. Wulff, Z. Kristallogr. - Cryst. Mater. 34 (1901) 449.

[16] V. Donnelly, J. McCaulley, Surf. Sci. 238 (1990) 34.

[17] L. Lymperakis, J. Neugebauer, Phys. Rev. B (2009) 79

[18] J.N. Baillargeon, K.Y. Cheng, S.L. Jackson, G.E. Stillman, J. Appl. Phys. 69 (1991 8025.

[19] D.D. Koleske, A.E. Wickenden, R.L. Henry, J.C. Culbertson, M.E. Twigg, J. Cryst. Growth 223 (2001) 466.

[20] D.D. Koleske, A.E. Wickenden, R.L. Henry, W.J. DeSisto, R.J. Gorman, J. Appl Phys. 1998 (1998) 84

[21] K. Harafuji, T. Tsuchiya, K. Kawamura, J. Appl. Phys. 96 (2004) 2501.

[22] E.V. Yakovlev, R.A. Talalaev, A.S. Segal, A.V. Lobanova, W.V. Lundin, E.E. Zavarin, M.A. Sinitsyn, A.F. Tsatsulnikov, A.E. Nikolaev, J. Cryst. Growth 310 (2008) 4862.

[23] B. Beaumont, S. Haffouz, P. Gibart, Appl. Phys. Lett. 72 (1998) 921.

[24] Z. Chen, N. Fichtenbaum, D. Brown, S. Keller, U.K. Mishra, S.P. Denbaars, S Nakamura, J. Electr. Mater. 37 (2008) 546

[25] R. Feenstra, Surf. Rev. Lett. 7 (2000) 601.

[26] P. Shields, C. Liu, A. Šatka, A. Trampert, J. Zúñiga-Pérez, B. Alloing, D. Haško, F. Uherek, W. Wang, F. Causa, D. Allsopp, Phys. Status Solidi C 8 (2011) 2334.

[27] M. Muller, P. Veit, F.F. Krause, T. Schimpke, S. Metzner, F. Bertram, T. Mehrtens, K. Muller-Caspary, A. Avramescu, M. Strassburg, A. Rosenauer, J. Christen, Nano lett. 16 (2016) 5340.

[28] R. Koester, J.-S. Hwang, D. Salomon, X. Chen, C. Bougerol, J.-P. Barnes, D.S. Le Dang, L. Rigutti, A. de Luna Bugallo, G. Jacopin, M. Tchernycheva, C. Durand, J. Eymery, Nano Lett. 11 (2011) 4839.

[29] J.E. Northrup, C.G. van de Walle, Appl. Phys. Lett. 84 (2004) 4322. 